\title{
COMPARAISON DE L'EFFICACITÉ DES ÉNANTIOMORPHES DE LA METHIONINE CHEZ LE POUSSIN
}

\author{
C. CALFT et Monique FEN]IRY \\ Station de Recherches avicoles, \\ Centre national de Recherches zootechniques Jouy-en-Josas (Seine-et-(ise)
}

\section{SOMMAIRE}

L'efficacité pour la croissance des énantiomorphes et du racémique de la méthionine a été comparée chez le poussin. Chacune des formes isomériques a été ajoutée à deux taux différents à un régime déficient en acides aminés soufrés.

A chaque dose considérée, les L-,D- et DI -, méthionines ont manifesté la même efficacité alimentaire, mesurée par l'indice de consommation. Tilles n'ont pas provoqué de différences significatives à la fois entre les gains de poids et entre les consommations des animaux.

L'utilisation par les animaux des acides aminés sous leur forme énantiomorphe $D$ et $\mathrm{I}$ a été largement étudiée au cours des dernières décades et les conclusions ont été rassemblées par BERG, I953 et I959. Il apparait qu'aucune loi ne permet de prévoir l'efficacité relative d'une forme par rapport à l'autre. Suivant la nature de l'acide aminé et les conditions nutritionnelles, la torme $\mathrm{D}$ est plus ou moins apte à promouvoir la croissance. Parfois elle ne présente aucune efficacité : c'est le cas de la D-lysine et l'un de nous a pu confirmer ce fait chez le poussin (CALET, rg60 a et b). Parfois elle possède un effet de croissance égale à la forme $\mathrm{L}$ : 1a méthionine est 1'un des rares acides aminés pour lequel cette opinion est généralement admise.

Pourtant dans d'autres domaines que la croissance, le comportement de la Det de la L-méthionine sont différents. Ainsi Èwarns et $a l$. ( 1963 ) viennent confirmer récemment une étude de Gibson et Wisemax (I95I) qui concerne l'absorption intestinale des deux formes. Pout ces auteurs la vitesse de passage de la D-méthionine à travers la paroi intestinale serait légèrement plus faible que celle de la L-méthionine.

A eux seuls, ces résultats ne permettent pas d'imputer une infériorité à la Dméthionine, comme l'ont fait Gordon et SizER (I955) car ils méritent d'être discutés.

Bien que le ralentissement du passage à travers la paroi intestinale de la forme $\mathrm{D}$ ait été clairement établi au moyen d'éléments marqués, on ne peut cependant pas en conclure que le coefficient de digestibilité de la I)-méthionine s'en trouve abaissé pour autant.

De la même manière on a reproché aux formes I) en général d'être toxiques. 
Or il convient de rappeler les études de GulinNo et al. (I956) qui mesurent la dose létale $5^{0}$ de la méthionine sous ses deux formes. La dose qui fait mourir la moitié des effectifs đu lot est moins élevée de $20 \mathrm{p}$. roo avec la L-méthionine qu'avec la Dméthionine. C'est dire que la toxicité aiguë de cette dernière est inférieure à celle de la première.

Les différences que nous venons de signaler peuvent laisser croire que les deux formes isomériques de la méthionine ne possèdent pas toujours la même efficacité. Il semble donc que ces études doivent être reprises afin de lever les doutes qui pèsent encore sur cette question. On peut d'ailleurs l'envisager sous un angle qui a été généralement négligé dans le cas de la méthionine et qui concerne l'effet des formes isomères sur la consommation de l'animal. Jusqu'à présent, la comparaison de l'efficacité des énantiomorphes a été effectuée en nourrissant les animaux ad libitum. Or, la quantité d'aliment consommé par chacun des lots peut être très différente. Nous avons eu l'occasion de nous en rendre compte dans le cas de la DL-lysine et de la L-1ysine offerte à dose moitié moindre (CAI.ET, Ig60 a). Lorsque la quantité de lysine augmente, l'écart entre les gains de poids des deux lots s'accentue. Bien que l'on soit tenté de conclure que la D-lysine s'oppose à l'utilisation de la L-lysine, un examen plus approfondi fait apparaître un effet concomitant de la D-1ysine sur la consommation d'aliment. Pour une dose de I,-lysine ingérée, la croissance est identique quelle que soit la forme de la lysine : le seul effet de la I)-1ysine est de réduire l'appétit de l'animal.

Dans le cas présent, nous avons voulu plus spécialement étudier le rôle éventuel de la D-méthionine sur la consommation du poussin. Pour cela nous avons eu recours à une méthode de rationnement que nous avons expérimentée depuis I96I (CALE'T, JOUANDET, BARATOU, Ig6I).

\section{MA'TÉRIEL, E'T MÉ'THODES}

Soixante-dix poussins males issus du croisement Rhode $\times$ Wyandolte sont habitués depuis leur naissance à vivre en cage individuelle. Au moment où débute l'expérience Io blocs de 7 poussins sont constitués d'après le poids des animaux et leur gain de poids antérieur. Les poussins sont alors âgés de 4 semaines et leur poids moyen est de $320 \mathrm{~g}$.

Les rations des sujets expérimentaux sont distribuées séparément. La première est représentée par un régime protidique enrichi en vitamines dont la composition centésimale est donnée dans le

TABLEAU I

Protocole experimental

\begin{tabular}{|c|c|}
\hline Lots & Régrimes \\
\hline $\begin{array}{l}\text { A } \\
\text { B } \\
\text { C } \\
\text { D } \\
\text { l: } \\
\text { F } \\
\text { G }\end{array}$ & $\begin{array}{l}\text { Régime protidique de base (R. I. I3.) } \\
\text { R.P.B. + } 1 \% \text { de DL-méthionine } \\
\text { R.P.B. }+1 \% \text { de D-méthionine } \\
\text { R.P.B. + } 1 \text { de L-méthionine } \\
\text { R.P.B. + } 0,3 \% \text { de DL-méthionine } \\
\text { R.P.B. }+0,3 \% \text { de D-méthionine } \\
\text { R.P.B. + } 0,3 \% \text { de I-méthionine }\end{array}$ \\
\hline
\end{tabular}


tableau 2. La méthionine, lorsqu'elle est étudiée, est ajoutée à ce régime à raison de o,3 ou I p. Ioo selon le protocole figurant au tableau $\mathrm{I}$. La seconde ration ne renferme que des éléments non azotés (voir sa composition au tableau 3). Les deux rations diffèrent en outre par leur mode de distribution. Elles sont offertes simultanément à l'animal mais le repas azoté est alloué en quantité limitée à raison de 5,2 g par animal et par jour ; le repas non azoté est consommé à volonté.

\section{TABLEAU 2}

Composition centésimale du régime protidique

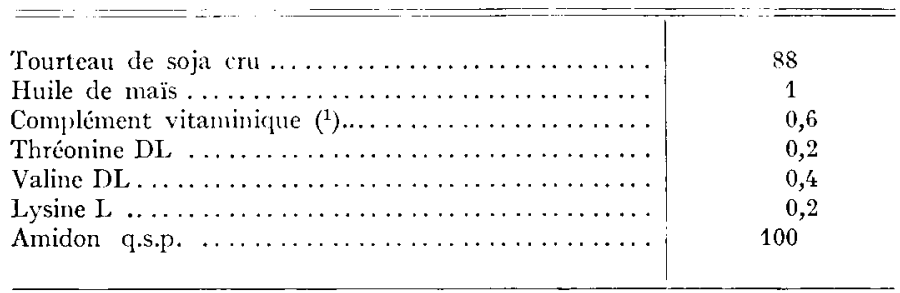

(1) Le complément vitaminique apporte par $100 \mathrm{~kg}$ de régime : Vitamine A : $1.600 .000 \mathrm{UI}$, Vitamine $D_{3}: 200.000 \mathrm{UI}$, Acétate d' $\alpha$-Tocophérol : $10 \mathrm{~g}$, 2-méthyl-1,4 naphtoquinone : $1 \mathrm{~g}$, Riboflavine : $3 \mathrm{~g}$, Pantothénate de calcium $3 \mathrm{~g}$, Amide de l'acide nicotinique $: 10 \mathrm{~g}$, Thiamine : $4 \mathrm{~g}$, Pyridoxine : $2 \mathrm{~g}$, Biotine : $0,12 \mathrm{~g}$, Acide folique : $0,6 \mathrm{~g}$, Cyanocobalamine : $0,01 \mathrm{~g}$, Acide ascorbique : $50 \mathrm{~g}$, Chlorure de choline : $25 \mathrm{~g}$.

\section{TABLEAU 3}

Composition centésimale de l'aliment non azoté

\begin{tabular}{|c|c|}
\hline Dextrose ........ & 55 \\
\hline 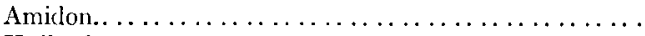 & 32,5 \\
\hline Huile de maïs $\ldots \ldots \ldots \ldots \ldots \ldots \ldots \ldots \ldots \ldots \ldots$ & 5 \\
\hline Cellulose $\ldots \ldots \ldots \ldots \ldots \ldots \ldots \ldots \ldots \ldots \ldots$ & 2 \\
\hline Complément minéral (1) . . . . . . . . . . . . . . & 5,5 \\
\hline
\end{tabular}

(1) Pour $100 \mathrm{~kg}$ d'aliment le complément minéral apporte les sels suivants exprimés en grammes:

\begin{tabular}{|c|c|}
\hline Carbonate de calcium & 1500 \\
\hline Phosphate dipotassique ......... & 730 \\
\hline Chlorure de Sodium $\ldots \ldots \ldots \ldots \ldots$ & 500 \\
\hline Sulfate de magnésium, $7 \mathrm{H}_{2} \mathrm{O} \ldots \ldots$ & 500 \\
\hline Phosphate bicalcique............ & 1700 \\
\hline Sulfate de manganese, $\mathrm{I}_{2} \mathrm{O} \ldots \ldots$ & 33 \\
\hline Citrate ferrique $\ldots \ldots \ldots \ldots \ldots$ & 33 \\
\hline Alun de potassium ........... & 1 \\
\hline Sélénite de sodium ............ & $0,0,5$ \\
\hline Bromure de sodium, $2 \mathrm{H}_{2} \mathrm{O} \ldots \ldots \ldots$ & 2,4 \\
\hline Chlorure de zine $\ldots \ldots \ldots \ldots \ldots \ldots$ & / \\
\hline Silicate de sodium. . . . . . . . . . & 5,5 \\
\hline Sulfate de cobalt, $7 \mathrm{H}_{2} \mathrm{O} \ldots \ldots \ldots$ & 0,2 \\
\hline Molybdate de sodium ............ & 0,9 \\
\hline Sulfate de cuivre, $5 \mathrm{II}_{2} \mathrm{O} \ldots \ldots \ldots$ & 2 \\
\hline Acide borique. $\ldots \ldots \ldots \ldots \ldots \ldots$ & 0,9 \\
\hline Iodure de potassium............. & i \\
\hline Amidon q.s.p. . . . . . . . . . . & 5500 \\
\hline
\end{tabular}


Signalons enfin que l'aliment azoté titre $40 \mathrm{p}$. 100 de matières azotées totales et 2650 calories métabolisables par $\mathrm{kg}$; la présence de soja cru et l'apport d'acides aminés libres non limitatifs tels que la lysine, la thréonine et la valine soulignent la carence de ce régime en méthionine. Le taux énergétique de l'aliment non azoté est de 3465 calories métabolisables par $\mathrm{kg}$.

L'expérience dure 3 semaines. Les poussins sont éclairés it heures par jour. La température de la pièce est maintenue voisine de $18^{\circ} \mathrm{C}$ et les poussins trouvent un complément de chaleur auprès d'une éleveuse individuelle. L'eau leur est accordée ad libitum.

\section{RÉSULTATS}

Pour la totalité de l'expérience, le tableau 4 présente les résultats portant sur le gain de poids, la consommation des divers régimes, la consommation totale et l'indice de consommation.

\section{TABLEAU 4}

Croissance ot consonmation d'aliment

(par animal et pour 2 I jours)

\begin{tabular}{|c|c|c|c|c|c|c|}
\hline \multirow[b]{2}{*}{ Lot: } & \multirow[b]{2}{*}{ Regrines } & \multirow[b]{2}{*}{$\begin{array}{l}\text { Gain de } \\
\text { poirts (ci) }\end{array}$} & \multicolumn{3}{|c|}{ Consonmuntion (g) } & \multirow{2}{*}{$\begin{array}{l}\text { Indice de } \\
\text { conson- } \\
\text { mation }\end{array}$} \\
\hline & & & $\begin{array}{l}\text { Rexinte } \\
\text { protirlicpue }\end{array}$ & $\begin{array}{l}\text { Niment } \\
\text { Hon aroté }\end{array}$ & $\begin{array}{l}\text { Consomma- } \\
\text { tion totale }\end{array}$ & \\
\hline$A$ & R.P.IS & $1,10, x$ & 253,7 & $x+2,5$ & $S i 30,2$ & $6,00)$ \\
\hline 13 & Méthioninc DL $1 "$ ". & 267,2 & $25 \times, k$ & $817, ;$ & 1076,1 & $4,0 \%$ \\
\hline C & Méthionine $\mathrm{D} 1 \mathrm{1}: \ldots$ & 299,9 & 258,0 & 579,5 & 1137,5 & 3,81 \\
\hline I) & Méthionins L 1 " & 280,2 & $257,{ }^{\prime}$ & $x=x, x^{\prime}$ & 1115,8 & 3,99 \\
\hline $\mathrm{l}:$ & Methionine DL $0, \because \quad 0 ;$. & 2335,7 & $25,2,2$ & $8: 39,8$ & 1098,0 & 4,67 \\
\hline $\mathrm{F}$ & Méthionine L 0,3 " & $21: 3,1$ & 257.1 & 768,1 & 1025,2 & $4,8 !$ \\
\hline G & Méthionine 1, $0.3 \%$. & 218,1 & $25(1,5$ & 5916,$1 ;$ & 1053,1 & 1,90 \\
\hline
\end{tabular}

La carence du régime protidique de base apparaît avec une grande netteté puisque le gain de poids du lot témoin est deux fois moindre que celui des lots les mieux supplémentés. En outre la plus faible addition de méthionine permet un accroissement de poids et une efficacité alimentaire augmentée de $60 \mathrm{p}$. Ioo.

Le problème important revient à distinguer, si on le peut, l'effet des 3 formes de méthionine selon leur dose. I e tableau 5 exprime les résultats des gains de poids sous forme statistique.

L'analyse de la variance montre que l'effet dî̀ à la dose est hautement significatif alors que l'effet dù à la forme isomérique ne l'est pas. Il existe par contre une interaction hautement significative entre la forme et la dose.

Ainsi l'addition de méthionine sous quelle forme que ce soit à la dose de 0,3 p. Ioo du régime protidique améliore considérablement l'efficacité du régime mais elle ne permet pas l'efficacité maximum. En effet, une addition supplémentaire de méthionine (jusqu'à I p. roo) provoque un bénéfice nouveau. 
Par ailleurs, chaque énantiomorphe et le racémique possèdent des efficacités égales puisqu'il est impossible de mettre en évidence une différence significative entre les 3 substances quelle que soit leur dose.

TABLEAU 5

Analyse de la variance

\begin{tabular}{|c|c|c|c|}
\hline $\begin{array}{l}\text { Origine de la } \\
\text { variation }\end{array}$ & $\begin{array}{l}\text { Degrés } \\
\text { de liberté }\end{array}$ & $\begin{array}{c}\text { Carré } \\
\text { nover }\end{array}$ & Signification \\
\hline Dose $\ldots \ldots \ldots \ldots \ldots \ldots$ & 1 & 51450 & H. S. \\
\hline Forme isomère ......... & 2 & 872 & N.S. \\
\hline Interaction dose $\times$ forme . & 2 & 6746 & H. S. \\
\hline Erreur................ & 54 & 774 & - \\
\hline
\end{tabular}

Enfin, la consommation d'aliment non azoté ou encore la consommation totale évolue dans le même sens que le gain de poids. Toutefois, au fur et à mesure que la dose de méthionine s'accroît, la nourriture ingérée augmente moins vite que le gain de poids et l'indice de consommation s'en trouve amélioré. Ce résultat s'exprime d'une manière plus analytique si l'on considère les valeurs du coefficient d'efficacité protidique et du coefficient d'efficacité énergétique qui figurent au tableau 6 . On remarque en conséquence que le rapport entre les calories librement consommées et les protides ingérés augmente avec l'efficacité du régime.

TABLEAU 6

Influence de la forme isomère de la méthionine sur l'efficacité du régime (pour la durée de l'expérience)

\begin{tabular}{|c|c|c|c|c|c|c|}
\hline Lots & $\begin{array}{l}\text { Gain de } \\
\text { poids }(g)\end{array}$ & $\begin{array}{c}\text { M. A. T. } \\
\text { consommées } \\
(g)\end{array}$ & $\begin{array}{l}\text { Gain de } \\
\text { poids } \\
\text { ML. A. T. } \\
\text { ingérées }\end{array}$ & $\begin{array}{l}\text { Calories } \\
\text { ingérées }\end{array}$ & $\begin{array}{l}\text { Gain de } \\
\text { poids } \\
\text { calories } \\
\text { ingérées }\end{array}$ & $\begin{array}{l}\text { Calories } \\
\text { ingérées }\end{array}$ \\
\hline A : régime de base .. & 140,8 & 101,3 & 1,39 & 2690 & 5,26 & 26,5 \\
\hline $\mathrm{B}:$ R.B. + DL $1 \%$. & 267,2 & 103,5 & 2,58 & 3509 & 7,62 & 33,9 \\
\hline $\mathrm{C}: \mathrm{R} . \mathrm{B} .+\mathrm{D} 1 \% \ldots$ & 299,9 & 103,3 & 2,90 & 3722 & 8,05 & 36,6 \\
\hline $\mathrm{D}: \mathrm{R} . \mathrm{B} .+\mathrm{L} 1 \% \ldots$ & 280,2 & 102,9 & 2,72 & 3648 & $7,7 / \mathrm{k}$ & 35,4 \\
\hline $\mathrm{E}:$ R.B. $+\mathrm{DL} 0,3 \%$ & 235,7 & 103,3 & 2,28 & 3592 & 6,61 & 34,8 \\
\hline $\mathbf{F}:$ R. B. + D $0,3 \%$. & 213,1 & 102,8 & 2,07 & 3341 & 6,38 & 32,5 \\
\hline $\mathrm{G}: \mathrm{R} . \mathrm{B} .+\mathrm{L} 0,3 \%$. & 218,1 & 102,6 & 2,12 & 3438 & 6,37 & 33,5 \\
\hline
\end{tabular}

Il est cependant utile de mentionner que les deux premiers critères d'efficacité n'évoluent pas exactement parallèlement. Nous avons superposé sur un même graphique les variations du C.E.P. et du coefficient d'efficacité calorique en fonction du rapport entre les calories et les protéines ingérées par les animaux. 
Au fur et à mesure que le rapport C/P s'accroit, les deux coefficients augmentent mais il convient de souligner que le taux d'accroissement du C.E.P. est plus élevé que celui du coefficient d'efficacité calorique. En outre, pour un même $\mathrm{C} / \mathrm{P}$, les régimes supplémentés par les plus forts taux de méthionine sont les plus efficaces pour les deux critères considérés.

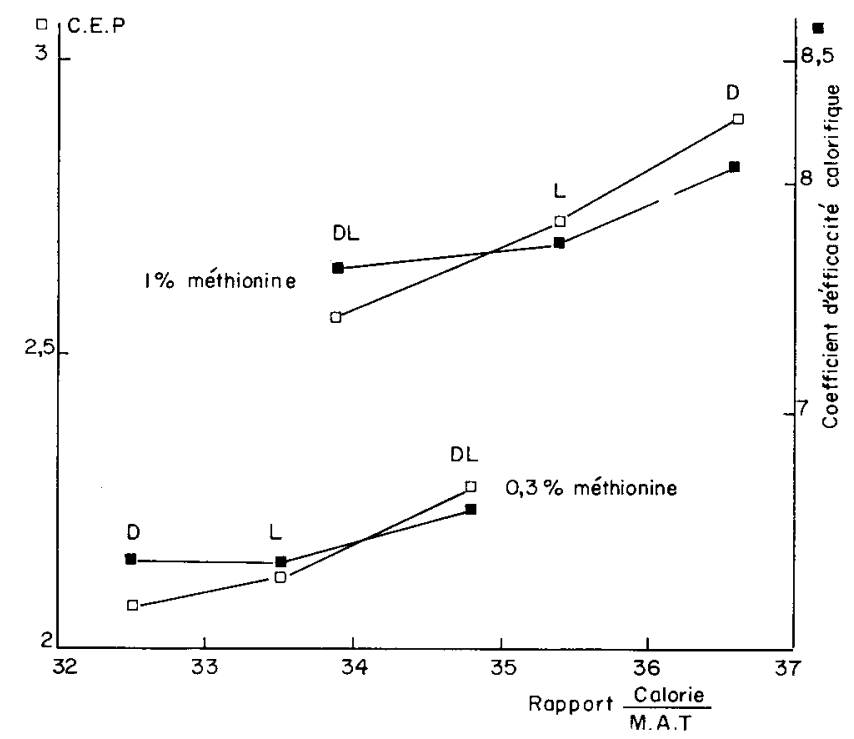

TiG. I. - Variations comparées des efficacités protidique et énergétique

\section{DISCUSSION}

La méthode que nous avons appliquée présente l'avantage de maintenir constante l'ingestion du facteur étudié sans entraver le poussin dans la satisfaction de son besoin énergétique. Le bien fondé de cette méthode est maintenant bien établi en France tant chez le poulet (CAI,ET et MeLot, I96I ; CalieT et BARATOU, I962 ; Calet et Albessard, ig63 ; Calet, Gulliaume, Deipech et Jacouot, I964) que chez le Rat par Henry et Rírat (I962). Enfin Peretianu et Abraham (I963) proposent de l'employer dans le contrôle pratique de la qualité des aliments du bétail.

Dans 1'expérience présente, nous confirmons des résultats précédemment établis. D'une part, l'élévation de la valeur biologique đu régime se traduit toujours par une augmentation de la quantité d'aliment consommé ; d'autre part, la séparation des constituants du régime en deux repas permet une amélioration simultanée des efficacités calorique et protidique. Nous retrouvons également que cette dernière s'accroît plus vite que la première lorsque la valeur biologique des protides devient de plus en plus élevée (Guillaume et CAL,ET, I964).

Nos conditions expérimentales permettent de révéler au mieux les différences 
d'efficacité des régimes. Dans le cas précis des formes isomériques de la méthionine, il n'apparaît aucune différence entre leur valeur alimentaire et ceci quelle que soit la dose étudiée. On peut penser que les doses utilisées sont excessives. En fait, nous les avons calculées en pourcentage du régime protidique qui ne représente qu'une partie de 1'ingéré total. En pourcentage de la consommation globale, les valeurs sont beaucoup plus faibles. Dans les lots B, C, D où la méthionine est apportée à raison de I p. Ioo d'aliment protidique, la consommation d'aliment azoté est de $258 \mathrm{~g}$ et la moyenne de la consommation totale est de I I Io g. Cela représente un taux protidique de 9,28 p. Ioo et un taux de méthionine supplémentaire de $0,23 \mathrm{p}$. Ioo. Dans les lots $\mathrm{E}, \mathrm{F}$ et $\mathrm{G}$ où la méthionine est fournie à raison de $0,3 \mathrm{p}$. roo de l'aliment protidique consommé à raison de $257 \mathrm{~g}$, la consommation moyenne totale est de I $059 \mathrm{~g}$. Cela revient à dire que le taux des protides dans la totalité des aliments ingérés est de $9,70 \mathrm{p}$. Ioo et celui de méthionine ajoutée est de $0,07 \mathrm{p}$. Ioo. Nous nous trouvons donc dans des limites tout à fait raisonnables qui sont celles utilisées généralement par d'autres auteurs. Ainsi nos travaux confirment les résultats de GrAU et Almouist (I943) qui attribuent la même efficacité à la $L_{\text {- }}$ et à la DL-méthionine chez le poulet. De même Ĺvinilié, Shapiro et Fisher (I960), étudient les besoins minimum du poussin en différents aminoacides et trouvent que pour la méthionine et la thréonine l'isomère $\mathrm{D}$ est complètement utilisé. Chez le Rat, WRETLLIND et Rose (I950), notent que 0,4 p. Ioo de méthionine couvrent aussi bien les besoins de l'animal lorsqu'ils sont représentés par la forme $\mathrm{I}$ ou la forme D. Mêmes conclusions plus récentes de BERG (I959), SAUBERLICH (I96I) et NJAA (I962), chez le Rat d'une part, et de Bruggemann, Drepper et Zucker (I962), chez le poussin. On peut imaginer au moins deux raisons au divorce entre les résultats de GoRDon et SIZER (I955), et ceux des autres auteurs, mais on ne peut guère les vérifier puisque les conditions expérimentales des premiers ne sont pas connues.

Une première raison pourrait être imputée à la composition des régimes. WRETLIND (I952) a montré que la D-méthionine équivaut à la $L$-méthionine lorsque dans le régime synthétique tous les acides aminés sont sous forme $L_{\text {. }}$ Au contraire la forme $\mathrm{D}$ est moins efficace si une partie des autres acides aminés sont des racémiques. Cette opinion vient d'être confirmée par MARRETT, BIRD et SUNDE (I964) chez des poussins nourris de régimes synthétiques lorsque les acides aminés sont soit en totalité sous forme $\mathrm{L}$, soit représentés par I $5 \mathrm{p}$. Ioo de forme $\mathrm{D}$. On peut admettre avec ces auteurs que la quantité de D-aminoacide oxydase dont dispose l'animal est suffisante pour effectuer la stéréonaturalisation d'un acide aminé mais qu'elle ne l'est plus lorsque la plupart des acides aminés du régime est constituée de racémique.

Une autre raison de la divergence peut provenir de la dose employée. On sait en effet que les doses élevées d'acides aminés sont préjudiciables à la croissance de l'animal (HARPER et al., I955). Or il se trouve que les animaux tolèrent moins bien ces fortes doses lorsque 1'on leur administre des formes D. STEKOL (Ig62) ajoute à 2 à $4 \mathrm{p}$. Ioo de méthionine un régime à $24 \mathrm{p}$. Ioo đe caséine. Dans ces conditions les doses excessives de D-méthionine sont plus toxiques que les mêmes quantités de I-méthionine et entraînent des lésions microscopiques et histologiques au niveau du foie et de la rate.

Lorsque l'on enrichit le régime avec des doses plus raisonnables de méthionine, dans le souci de remédier à leur déficit en acides aminés soufrés, de tels désavantages ne sont pas à craindre. Dans nos essais, comme dans de nombreux autres où des 
conditions compatibles avec la pratique ont été respectées, la méthionine se révèle un puissant agent de croissance du poussin aussi bien lorsqu'elle est employée sous l'une ou l'autre forme isomérique.

Reģu pour publication en septembre 1965.

\section{SUMMARY}

COMPARISON OF THE EFFICIENCY OF ENANTIOMORPHS OF METHIONINE FOR CHICKENS

The efficiency of different forms of methionine for growth of chickens was studied. A method of feeding previously described by Calet and MeLot (I96I) for chickens and by Peretianu and AbrahaM (1963) for rats was used. For it a limited ration of protein and a protein-free feed are offered separately but at the same time. The protein feed consists mainly of crude soya which is deficient in sulphur amino acids. Methionine is added to that feed as the L-, D- or DL-forms, each as 0.3 or I.o p. I00. At the end of three weeks weight gain and intake of protein-free feed are recorded.

Results showed that the amount of methionine given had a highly significant effect. With neither amount was it possible to show any significant difference in growth or intake of feed attributable to the different isomers. In the same way, at either level the efficiency of feed utilization was the same for the three forms of methionine.

\section{RÉFÉRENCES BIBLIOGRAPHIQUES}

Berg C. P., 1953. Physiology of the D-amino acids. Physiol. Rei', 33, $145-189$.

Berc C. P., I959. Utilization of D-amino acids. In "Protein and amino acid nutrition". Academic Press, New York, Albanese A. A. ed. London 1959, 57-96,

BruggemanN J. Drepper K. ZuCker II. I962. Quantitative Bestinmung der verwertung von D-, I-, DL -Methionin und Ca-DL, 2 Hydroxy-4-methylthiobutyrat durch das IIühnerküken. Naturaissenschaften 49, 334.

Calet C., I96o a. Comparaison de l'efticacité pour la croissance du poussin des isomères D- et L- de la lysine (abstr.). V Intern. Congr. Vutr., September 1-7, 1960; Washington D. C., 60.

CaLeT C., $1960 b$. Contribution it l'étude de l'efficacité pour la croissance du poussin des formes L ct DI. de la lysine. Ann. Natr. Alim., 14, 28;-290.

Calet C., Joundet C., Baratot J., I96I. Variation de la consommation spontanée d'énergie du poussin en fonction de la nature des matières azotées du régime. Inn. Biol. anim. Bioch. Biophys., 1, 5-9.

Calet C., Melot M., Ig6r. Eifficacité comparée pour la croissance du poussin de la méthionine et du sel de calcium de l'acide hydroxyméthylthiobutyrique (II. H. A.). Ann. Zoolech., 10, 205-213.

Calet C., Abrainam F., Baratov J., ig62. Influence de la valeur biologique des protides du régime sur la consommation spontanée d'énergie du poussin. 7th World's Poult. Congr., Sydney, p. 208-2 I I.

Calet C., Albessard A., I963. Influence du mode de distribution des matières azotées du régime sur l'efficacité et le comportement alimentaire du poussin. Inn. Biol. anim. Bioch. Biophys., 3, 353-367.

Calet C., Guillaume J., Delpecie P., Jacelot R., rg 64 . Le róle des protides dans l'utilisation des nutriments énergétiques chez le poussin. C. R. Acad. Sci., 258, 3104-3106.

EDWARDS C. H., Gadsden F. L., EDwards G. A., 1963. Utilization of methionine by the adult rat. II. Absorption and tissue uptake of $\mathrm{L}_{-}$- and DL-methionine. $J$. Nutr., 80, 69-74.

Gibson Q. H., Wisemix G., 195r. Selective absorption of steren-isomers of amino acids from loops of the small intestine of the rat. Biochem. J., 48, 426-429.

Gordon R. S., SIzER I. W., 1955. The biological equivalence of methionine hydroxy analogue (abstr.) Poult. Sci., 34, 1198.

Grau C. R., Almolist I. J, 1943. Ltilization of the sulfur amino acids by the chick. J. Nuir, 26, $631-640$.

Gullaume J., Calet C., r964. Le rôle des protides dans l'utilisation des aliments du poussin. I. Limites supérieures de l'efficacité azotée et énergétique mesurée à partir de la composition corporelle de l'animal (note prélininaire). Ann. Biol. anim. Bioch. Biophys., 4, 63-68. 
Gulino 1’., Winitz M., Birnbaum S. M., Cornfield J., Otey M. C., Greenstein J. P., i956. Studies on the metabolism of amino acids and related compounds in vivo. I. Toxicity of essential amino acids, individually and in mixtures, and the protective effect of L-arginine. Arch. Biochem. Biophys, 64, 319-3.32

Harper A. E., 1958, Balance and imbalance of amino acids. Ann. N. Y. Acad. Sci., 69, 1025.

IIENRY Y., RérAT A., I962. Influence de la qualité et de la quantité des matières azotées ingérées sur la consommation spontanée d'énergie chez le rat en croissance. Ann. Biol. anim. Bioch. Biophys., 2, $267-276$.

Léveilité (i. A., Silapiro R., Fisher H., ig6o. Amino acid requirements for maintenance in the adult rooster. IV. The requirements for methionine, cystine, phenylalanine, tyrosine and tryptophin ; the adcquacy of the determined requirements. J. Nutr., 72, 8-1 5.

Marrett L., Bird H. R., Sunde M. L., I964. The effects of different isomers of methionine on growth of chicks fed amino acid diets. Poull. Sci., 43, i i I 3 - I I 8 .

NJAA L. R., I962. Utilization of methionine sulphoxide and methionine sulphone by the young rat. Bril. J. Nutr., 16, 57 I-577.

Perretanu J., Abraham J., i963. Nouvelle technique de mesure du coefficient d'efficacité protéique. Ann. Nutr. Alim., 17, 81-IO2.

SAUbericir H. E., I $96 \mathrm{r}$. Effect of vitamin $B_{6}$ on the growth of rats fed diets limiting in an essential amino acid and on the utilization of isomers of tryptophan, methionine and valine. J. Nutr., 74, $289-297$.

Stekol J. A., Szaran J., 1962. Pathological effects of excessive methionine in the diet of growing rats J. Nutr., 77, 8I-90.

WRETIIND K. A. J., I952. The availability for growth and the toxicity of L- and D-phenylalanine. Acla. Physiol. Scand., 25, 276-285.

WRETInd K. A. J., Rose W. C., I950. Methionine requirement for growth and utilization of its optical isomers. J. Biol. Chem., 187, 697-703. 